TTP00-22

hep-ph/0010217

October 2000

\title{
Kinematic Effects in Radiative Quarkonia Decays
}

\author{
STEFAN WOLF \\ Institut für Theoretische Teilchenphysik, Universität Karlsruhe, \\ D-76128 Karlsruhe, Germany
}

\begin{abstract}
Non-relativistic QCD (NRQCD) predicts colour octet contributions to be significant not only in many production processes of heavy quarkonia but also in their radiative decays. We investigate the photon energy distributions in these processes in the endpoint region. There the velocity expansion of NRQCD breaks down which requires a resummation of an infinite class of colour octet operators to so-called shape functions. We model these non-perturbative functions by the emission of a soft gluon cluster in the initial state. We found that the spectrum in the endpoint region is poorly understood if the values for the colour octet matrix elements are taken as large as indicated from NRQCD scaling rules. Therefore the endpoint region should not be taken into account for a fit of the strong coupling constant at the scale of the heavy quark mass.
\end{abstract}

PACS Nos.: 11.10.St, 12.39.Jh, 13.25.Gv 


\section{Introduction}

Since the discovery of the $J / \psi$ [目] and the $\Upsilon$ [2] heavy quarkonia decays are one of the most interesting laboratories for investigations within the framework of perturbative QCD. In particular these bound states of a heavy quark $Q$ and its antiparticle $\bar{Q}$ have been examined to extract the value of the strong coupling constant $\alpha_{s}$ at the scale of the heavy quark mass $m_{Q}$.

Early theoretical analyses starting from the calculation of the total rates in leptonic and inclusive hadronic decays [3] were done in the colour singlet model (CSM). It assumes the quark-antiquark pair being in the same quantum state $n={ }^{2 S+1} L_{J}^{(C)}$ on the partonic level as the corresponding quarkonium on the hadronic level. In particular the $Q \bar{Q}$ pair has to be in a colour singlet state $(C=1)$ when it annihilates. As a consequence of this requirement the underlying partonic process in the radiative decay $H \rightarrow \gamma X$ of a quarkonium $H$ in the ground state ${ }^{3} S_{1}$ is the annihilation of the heavy $Q \bar{Q}$ pair into a photon and at least two gluons. This process was calculated first in [4].

Great theoretical progress in the understanding of bound states of heavy $Q \bar{Q}$ systems has been achieved by NRQCD (Non-Relativistic Quantum Chromo-Dynamics) [5]. In this theory quarkonia decays are factorized into two step processes: the short-distance annihilation of a $Q \bar{Q}$ pair with fixed total spin $S$, orbital angular momentum $L$, and total angular momentum $J$ and its preceding long-distance transition into this state. While the partonic subprocess can be calculated perturbatively to definite order in $\alpha_{s}$ the nonperturbative subprocess $H \rightarrow Q \bar{Q}[n]+$ soft degrees of freedom is parameterized by NRQCD matrix elements. They are the NRQCD counterparts of the wave function at the origin in the colour singlet model and give the probability for finding the quark-antiquark pair in the quantum state $n$ at the moment of annihilation. In principle the values of these parameters are unknown and must be fitted to experimental data [6] or computed on the lattice [7]. Nevertheless NRQCD provides scaling rules which e.g. predict colour octet matrix elements being suppressed by powers of the non-relativistic velocity $v$ with respect to the leading order colour singlet matrix element. This typical velocity of the heavy (anti)quark inside the quarkonium simultaneously serves as expansion parameter of the effective field theory. As a result NRQCD describes a decay rate by an infinite sum over matrix elements of four fermion operators with Wilson coefficients which on their part are expansions in $\alpha_{s}$.

Taking only the leading order in $v / c$ the NRQCD result coincides with the one of the CSM. However, subleading terms in the velocity expansion could be still important numerically: In radiative decays the partonic kernel of a colour octet contribution is enhanced by an inverse power of $\alpha_{s}\left(m_{Q}\right)$ with respect to the leading order colour singlet mode. While the leading term needs two hard gluons in the final state (cf fig. 11(a)) a $Q \bar{Q}$ pair in a colour octet state can annihilate into a photon and a single gluon (cf fig. 1(b)). Thus one may also take into account the subleading terms in $v / c$.

The photon energy spectrum in the hard subprocess $Q \bar{Q}[n] \rightarrow \gamma X$ of radiative decays has been calculated in next-to-leading order perturbative QCD for both the colour singlet mode [8] and the colour octet modes [9]. Another perturbative contribution may become important in the upper endpoint region $\left(z=2 E_{\gamma} / M_{H} \rightarrow 1\right)$ of the spectrum. Due to an 


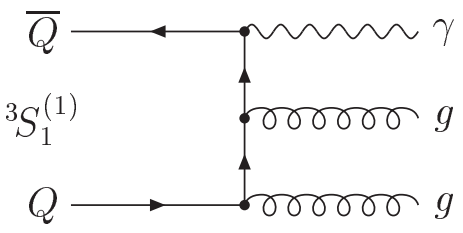

(a)

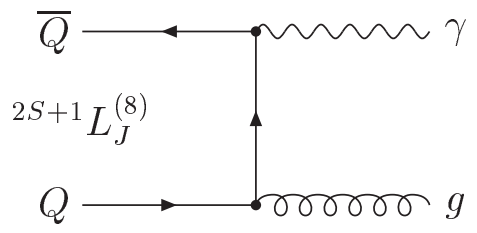

(b)

Figure 1: Direct contributions to the radiative decay of a $Q \bar{Q}$ pair: on the left (a) one of six colour singlet diagrams, on the right (b) one of two diagrams per colour octet mode.

imperfect cancellation between terms stemming from real and virtual emission of soft gluons one could expect potentially large logarithms $\ln (1-z)$ to all orders of the perturbation theory. A resummation of these logarithms would then give rise to a Sudakov suppression $\sim \exp \left(-\alpha_{s} \ln ^{2}(1-z)\right)$. Though an earlier analysis [10 claimed such a Sudakov damping factor in the colour singlet mode a more recent work [11] predicts such Sudakov factors in the colour octet channels only while the logarithms should cancel order by order $\alpha_{s}$ in the colour singlet mode.

Besides these perturbative contributions several non-perturbative effects have been investigated as well. They become important near the phase space boundaries where the photon energy fraction $z$ is small or close to 1 , respectively. For low values of $z$ there is a large fragmentation contribution caused by the collinear emission of a photon from a light (anti)quark in the final state. Examples for such processes are diagrammed in fig. 2. They have been investigated in [12 for the colour singlet mode and in [9] for the colour octet channels.

At the upper endpoint of the spectrum two different sources for non-perturbative effects exist. The first one is the phase space effect associated with the hadronization of massless gluons into massive final states. This effect usually is considered by a parton shower Monte Carlo thereby generating a non-zero invariant mass for the outgoing gluon(s) [13. Another method based on the introduction of an effective gluon mass [14] could obtain the appropriate phase space suppression by fitting the values of the effective gluon mass to data of radiative $J / \psi$ [15] and $\Upsilon$ [16, 17] decays independently [18].

In this article we concentrate on another non-perturbative effect contributing to the upper endpoint of the spectrum. In this region NRQCD operators connected to the centerof-mass (cms) movement of the $Q \bar{Q}$ pair inside the quarkonium could become significant even though they are subleading in the sense of the naive NRQCD power counting [19, 20]. It has been shown in [20] explicitly that the NRQCD velocity expansion breaks down near the endpoint. The reason for this breakdown is the kinematical enhancement of the $\mathrm{cms}$ operators. In the endpoint region the expansion parameter is $v^{2} / \epsilon$ rather than $v^{2}$ where $\epsilon=1-E_{\gamma} / m_{Q}$ is a measure for the distance from the endpoint. Thus the velocity expansion works fine only for photon energies that are significantly further away from the endpoint than $\Delta E_{\gamma} \sim m_{Q} v^{2}$. However, the range of applicability of NRQCD can be extended to higher values for $E_{\gamma}$ by the resummation of an infinite class of operators into so-called shape 


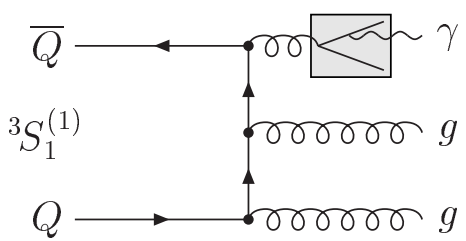

(a)

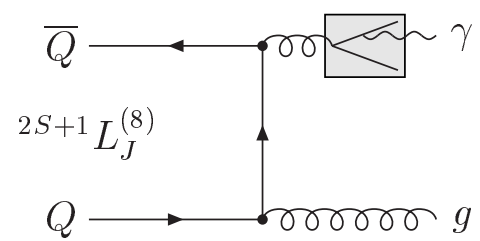

(b)

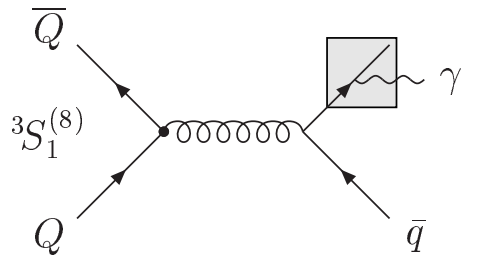

(c)

Figure 2: Fragmentation contributions to the radiative decay of a $Q \bar{Q}$ pair: colour singlet contribution (a), colour octet contribution (b) and (c). The non-perturbative subprocesses inside the grey boxes are described by the gluon $(\mathrm{a})+(\mathrm{b})$ and the quark (c) fragmentation function respectively.

functions [20]. Thereafter the shape function improved spectrum holds up to a resolution of $\Delta E_{\gamma} \sim m_{Q} v^{2}$.

The shape function formalism yields different shape functions for the different quantum states $n$ of the quark-antiquark pair $(Q \bar{Q}[n])$. In the leading colour singlet case the shape function is nothing else than a number namely the corresponding NRQCD matrix element. In the colour octet modes the shape functions are real functions reflecting the phase space dependence of the soft gluon radiation. Thus the partonic spectrum of the relevant channels $n=\left\{{ }^{1} S_{0}^{(8)},{ }^{3} P_{J}^{(8)},{ }^{3} S_{1}^{(8)}\right\}$ which is proportional to $\delta\left(E_{\gamma}-m_{Q}\right)$ is smeared out to a quite broad peak.

As mentioned above their contribution may be as large as the one from the leading order. If we compare the total rates of the leading colour singlet and a subleading colour octet term we get

$$
\Gamma_{1}: \Gamma_{8}=\frac{\alpha_{s}\left(m_{Q}\right)}{4 \pi}: v^{4}=\mathcal{O}(1)
$$

Thus it is worth to model the non-perturbative shape functions to estimate their influence on the photon energy distribution. For the construction of the model we keep close to a model successfully used for shape functions in quarkonium production [21]. In this case the shape functions which originally were defined in [22] have been modeled by soft gluon emission from the final state. Inspired by the success in describing the $J / \psi$ production in $B$ decays and in the photoproduction channel we take over the physically simple picture of radiating off soft gluons from the heavy (anti)quark.

We will proceed as follows: First we will recapitulate the result of underlying partonic process $Q \bar{Q}[n] \rightarrow \gamma+g$ and $Q \bar{Q}[n] \rightarrow \gamma+g+g$ for $n={ }^{2 S+1} L_{J}^{(C)} \in\left\{{ }^{1} S_{0}^{(8)},{ }^{3} P_{J}^{(8)},{ }^{3} S_{1}^{(8)}\right\}$ and $n={ }^{3} S_{1}^{(1)}$ respectively. Moreover we will show to what extent fragmentation contributions must be taken into account. Afterwards the construction and application of our shape function model in the decay mode is given. Finally we discuss the results of the numerical evaluation of the semi-inclusive decay $\Upsilon(1 \mathrm{~S}) \rightarrow \gamma+$ light hadrons. 


\section{The partonic calculation}

Within NRQCD the photon energy spectrum in the semi-inclusive decay $H \rightarrow \gamma X$ of a quarkonium is represented by the operator product expansion

$$
\frac{d \Gamma}{d \hat{z}}=\sum_{n} \mathcal{C}[n]\langle H|\mathcal{O}[n]| H\rangle
$$

Here the Wilson coefficient $\mathcal{C}[n]$ is calculable perturbatively and gives the differential rate $d \Gamma_{n} / d \hat{z}$ in the decay of a $Q \bar{Q}$ pair with quantum numbers $n$ into a photon and light hadrons $X$. Note that in conventional NRQCD the photon energy is normalized on the quark rather than on the quarkonium mass: $\hat{z}=E_{\gamma} / m_{Q}$.

\subsection{Direct contributions}

Equation (2) includes not only the contributions from the direct production of a photon (fig. 目(a)) but also the production via fragmentation (fig. 目(b)). We will come to this point later. We first deal with the direct channels. The leading term in the non-relativistic expansion is the colour singlet mode displayed in diagram 1(a). It has been calculated perturbatively up to $\mathcal{O}\left(\alpha_{s}\right)$ [8]. For the sake of simplicity we restrict ourselves on the tree level contribution. It is [ـ]

$$
\begin{aligned}
\mathcal{C}_{\gamma}^{\operatorname{dir}}\left[{ }^{3} S_{1}^{(1)}\right](\hat{z})=\frac{32 e_{Q}^{2} \alpha_{\mathrm{em}}\left(m_{Q}\right) \alpha_{s}^{2}\left(m_{Q}\right)}{27 m_{Q}^{2}} & {\left[\frac{2-\hat{z}}{\hat{z}}+\frac{\hat{z}(1-\hat{z})}{(2-\hat{z})^{2}}\right.} \\
+2 \frac{1-\hat{z}}{\hat{z}^{2}} & \left.\ln (1-\hat{z})-2 \frac{(1-\hat{z})^{2}}{(2-\hat{z})^{3}} \ln (1-\hat{z})\right] .
\end{aligned}
$$

The subleading terms $\mathcal{O}\left(v^{4}\right)$ in the velocity expansion arise from Feynman diagrams like fig. 1 $1(\mathrm{~b})$, where ${ }^{2 S+1} L_{J}^{(C)}={ }^{1} S_{0}^{(8)},{ }^{3} P_{0}^{(8)}$ or ${ }^{3} P_{2}^{(8)}$. They are perturbatively enhanced in comparison to the colour singlet channel $\left(\mathcal{O}\left(\alpha_{s}\right)\right.$ versus $\left.\mathcal{O}\left(\alpha_{s}^{2}\right)\right)$. Due to their two body kinematics the photon spectrum is fixed to a definite energy value:

$$
\mathcal{C}_{\gamma}^{\operatorname{dir}}[n](\hat{z})=\frac{1}{2 \cdot 2 m_{Q}} \frac{1}{8 \pi} H[Q \bar{Q}[n] \rightarrow \gamma g]\left(2 m_{Q}\right) \delta(1-\hat{z}) .
$$

The spin and colour averaged squares $H[Q \bar{Q}[n] \rightarrow \gamma g]\left(2 m_{Q}\right)$ of the amplitudes for the three relevant channels are (again we take only leading terms in $\alpha_{s}$ ) [9]:

$$
\begin{aligned}
& H\left[Q \bar{Q}\left[{ }^{1} S_{0}^{(8)}\right] \rightarrow \gamma g\right]\left(2 m_{Q}\right)=\frac{256 \pi^{2} e_{Q}^{2} \alpha_{\mathrm{em}}\left(m_{Q}\right) \alpha_{s}\left(m_{Q}\right)}{2 m_{Q}} \\
& H\left[Q \bar{Q}\left[{ }^{3} P_{0}^{(8)}\right] \rightarrow \gamma g\right]\left(2 m_{Q}\right)=\frac{768 \pi^{2} e_{Q}^{2} \alpha_{\mathrm{em}}\left(m_{Q}\right) \alpha_{s}\left(m_{Q}\right)}{2 m_{Q}} \\
& H\left[Q \bar{Q}\left[{ }^{3} P_{2}^{(8)}\right] \rightarrow \gamma g\right]\left(2 m_{Q}\right)=\frac{1024 \pi^{2} e_{Q}^{2} \alpha_{\mathrm{em}}\left(m_{Q}\right) \alpha_{s}\left(m_{Q}\right)}{5 \cdot 2 m_{Q}} .
\end{aligned}
$$




\section{$2.2 \quad$ Fragmentation contributions}

Let us turn to the fragmentation contributions now. The corresponding processes are associated with diagrams like the ones in fig. 2. There the photon does not stem directly from the annihilation of the heavy quark-antiquark pair but from the fragmentation of a gluon or a light (anti)quark in the final state. Nevertheless we can keep the form of equation (2) to describe these contributions, since this subprocess is independent from the initial state effects parameterized in the NRQCD matrix elements. The Wilson coefficient is then obtained by the folding

$$
\mathcal{C}_{\gamma}^{\text {frag }}[n](\hat{z})=\sum_{a=g, q, \bar{q}} \int_{\hat{z}}^{1} \frac{d \hat{x}}{\hat{x}} \mathcal{C}_{a}^{\operatorname{dir}}[n]\left(\hat{x}, \mu^{2}\right) D_{a \rightarrow \gamma}\left(\hat{z} / \hat{x}, \mu^{2}\right)
$$

of the fragmentation function $D_{a \rightarrow \gamma}$ and the coefficient $\mathcal{C}_{a}^{\operatorname{dir}}[n]$ which is the perturbative part of the NRQCD decay rate of a $Q \bar{Q}$ pair with quantum numbers $n$ into a particle $a \in\{g, q, \bar{q}\}$ and other light degrees of freedom. The integration variable $\hat{x}$ indicates the energy of the particle $a$ normalized on the heavy quark mass: $\hat{x}=E_{a} / m_{Q}$.

Since the fragmentation takes place at a scale far below the heavy quark mass $m_{Q}$ it can be factorized from the hard subprocess. This is denoted by the factorization scale $\mu$ in (6). As usual one may derive a renormalization group equation from the $\mu$ independence of $\mathcal{C}_{\gamma}^{\text {frag }}[n]$ to shift potentially large logarithms between $\mathcal{C}_{a}^{\operatorname{dir}}[n]$ and $D_{a \rightarrow \gamma}$.

Though the fragmentation functions are non-perturbative objects a naive estimate for their order of magnitude is obtained from counting coupling constants and collinear singularities coming up in a perturbative calculation. For that we look at the subprocesses highlighted by grey boxes in fig. 2. The coupling of the photon to a light (anti)quark is proportional to $\alpha_{e m}\left(m_{Q}\right)$. For $D_{q \rightarrow \gamma}$ the leading term comes from the kinematic region where the photon and the (anti)quark are collinear. Thus one gets a factor $\ln \left(Q^{2} / Q_{0}^{2}\right)$ where $Q^{2} \sim m_{Q}^{2}$ from the phase space integration with a collinear cut-off parameter $Q_{0}$ of order $\Lambda_{\mathrm{QCD}}$. Accordingly one gets $\alpha_{e m}\left(m_{Q}\right) \alpha_{s}\left(m_{Q}\right) \ln ^{2}\left(Q^{2} / Q_{0}^{2}\right)$ for the gluon fragmentation function. Here the logarithm appears quadratically because quark, antiquark, and photon can become collinear simultaneously in this case.

The logarithms $\ln \left(Q^{2} / Q_{0}^{2}\right)$ could become so large that they could compensate the perturbative $\alpha_{s}$ suppression and thus confuse the perturbation series dramatically. This is seen in a easy way from the running of the strong coupling constant. At leading order the renormalization group equation yields

$$
\alpha_{s}\left(\mu^{2}\right)=\frac{\alpha_{s}\left(\mu_{0}^{2}\right)}{1+\alpha_{s}\left(\mu_{0}^{2}\right) \frac{\beta_{0}}{4 \pi} \ln \left(\frac{\mu^{2}}{\mu_{0}^{2}}\right)}
$$

with $\beta_{0}=\left(33-2 n_{f}\right) / 3$ if $n_{f}$ fermions are active. As long as $\mu$ is far above a typical hadronic scale $\mu_{0} \sim \Lambda_{\mathrm{QCD}}$ one can neglect the constant in the denominator. Hence

$$
\alpha_{s}\left(\mu^{2}\right) \ln \left(\mu^{2} / \mu_{0}^{2}\right) \sim \mathcal{O}(1)
$$


Based on this relation we receive fragmentation contributions with magnitudes comparable to the direct ones. This become clear if we rewrite the fragmentation functions in the leading logarithmic approximation. Then they have the form [23]

$$
D_{a \rightarrow \gamma}\left(\xi, Q^{2}\right)=\frac{2}{\beta_{0}} \frac{\alpha_{\mathrm{em}}\left(Q^{2}\right)}{\alpha_{s}\left(Q^{2}\right)} f_{a}(\xi)
$$

where $f_{a}$ is a phenomenological function of the fraction $\xi=E_{\gamma} / E_{a}$ of the photon and the parton energy. In case of comparable functions $f_{a}$ for the different partons $a$ the $\alpha_{s}$ in the denominator cancels the additional $\alpha_{s}$ in the hard subprocess of the fragmentation contributions.

Instead of using fitted functions for $f_{a}$ we will take the perturbative result for the fragmentation functions $D_{a \rightarrow \gamma}$. They are known in next-to-leading order [24] but we will restrict ourselves on the leading order for consistency with the investigation of the annihilation subprocess. The scale dependence of $D_{g \rightarrow \gamma}$ and $D_{q \rightarrow \gamma}$ is given by the leading order DGLAP equations [25, 26]

$$
\begin{aligned}
& \mu^{2} \frac{\partial}{\partial \mu^{2}} D_{q \rightarrow \gamma}\left(\xi, \mu^{2}\right)=\frac{e_{q}^{2} \alpha_{\mathrm{em}}\left(\mu^{2}\right)}{2 \pi} P_{q \rightarrow \gamma}(\xi), \\
& \mu^{2} \frac{\partial}{\partial \mu^{2}} D_{g \rightarrow \gamma}\left(\xi, \mu^{2}\right)=\frac{\alpha_{s}\left(\mu^{2}\right)}{2 \pi} \int_{\xi}^{1} \frac{d \eta}{\eta} P_{g \rightarrow q}(\eta) D_{q \rightarrow \gamma}\left(\xi / \eta, \mu^{2}\right)
\end{aligned}
$$

where $e_{q}$ is the charge of the light quark measured in units of the elementary charge. The Altarelli-Parisi splitting functions are [26]:

$$
P_{q \rightarrow \gamma}(\xi)=\frac{1+(1-\xi)^{2}}{\xi}, \quad P_{g \rightarrow q}(\xi)=\frac{1}{2}\left[\xi^{2}+(1-\xi)^{2}\right] .
$$

Integrating equation (10a) one obtains the quark fragmentation function

$$
D_{q \rightarrow \gamma}\left(\xi, \mu^{2}\right)=\frac{e_{q}^{2} \alpha_{\mathrm{em}}\left(\mu^{2}\right)}{2 \pi} P_{q \rightarrow \gamma}(\xi) \ln \left(\frac{\mu^{2}}{\mu_{0}^{2}(1-\xi)^{2}}\right)+D_{q \rightarrow \gamma}\left(\xi, \mu_{0}^{2}\right) .
$$

The $\xi$ dependence of the starting value $D_{q \rightarrow \gamma}\left(\xi, \mu_{0}^{2}\right)$ for the evolution in the factorization scale $\mu$ is mainly determined by a logarithm $\ln \left(1 /(1-\xi)^{2}\right)$ originating from the phase space integration [27]. It is already separated in (12). The remaining rest term cannot be calculated perturbatively. Therefore it has to be modeled, e.g. with a vector dominance model, or fitted to data. The latter was done by the ALEPH collaboration in a measurement of the $\gamma+(1$ jet $)$ rates for $\xi>0.7$ [28]. They get the best fit for a constant rest term $C=-1-\ln \left(M_{Z}^{2} /\left(2 \mu_{0}^{2}\right)\right)$ in

$$
D_{q \rightarrow \gamma}\left(\xi, \mu^{2}\right)=\frac{e_{q}^{2} \alpha_{\mathrm{em}}\left(\mu^{2}\right)}{2 \pi}\left[P_{q \rightarrow \gamma}(\xi) \ln \left(\frac{\mu^{2}}{\mu_{0}(1-\xi)^{2}}\right)+C\right] .
$$


The non-perturbative scale $\mu_{0}$ extracted from data is then

$$
\mu_{0}=0.14_{-0.12}^{+0.43} \mathrm{GeV} \Rightarrow C=-13.26_{-3.89}^{+2.81} .
$$

The non-perturbative piece of the gluon fragmentation function $D_{g \rightarrow \gamma}$ cannot be determined experimentally. For the sake of simplicity we will set $D_{g \rightarrow \gamma}\left(\xi, \mu_{0}\right)=0$. The leading quadratic logarithmic term reads

$$
D_{g \rightarrow \gamma}\left(\xi, \mu^{2}\right)=\frac{\alpha_{s}\left(\mu^{2}\right)}{2 \pi} \int_{\xi}^{1} \frac{d \eta}{\eta} P_{g \rightarrow q}(\eta) D_{q \rightarrow \gamma}\left(\xi / \eta, \mu^{2}\right) \frac{1}{2} \ln \left(\frac{\mu^{2}}{\mu_{0}^{2}}\right) .
$$

Since the experimental investigation of the photon spectrum in radiative quarkonia decays is limited to $z>0.4$ due to large uncertainties for soft photons caused by $\pi^{0}$ decays we need the fragmentation functions for $\xi>0.4$ only. In this region the contribution of the gluon fragmentation function is negligible. However, the quark fragmentation into a photon is significant for $\xi \sim 0.4$. Furthermore possibly large contributions in the endpoint region we are especially interested in are caused by the logarithmic divergence $\ln \left(1 /(1-\xi)^{2}\right)$.

At this stage one comment is in order. Since our approximation for the fragmentation functions and in particular relation (8) holds the better the larger the scale $\mu$ is, i.e. the larger the heavy quark mass $m_{Q}$ is, the leading log approximation is inaccurate or even not reliable for $m_{Q}=m_{c}$. Therefore we will concentrate our numerical investigation on the $\Upsilon$ decay as long as we do not restrict ourselves on large values for $z$.

Finally we need the perturbative results for the coefficient $\mathcal{C}_{a}^{\operatorname{dir}}[n]$ in (6). Again we distinguish between colour singlet and colour octet contributions. The calculation of the colour singlet mode (fig. 2(a) without the fragmentation subprocess) is, except for colour factors, the same as the decay rate of ortho-positronium [29]. Similarly the coefficient $\mathcal{C}_{a}^{\operatorname{dir}}\left[{ }^{3} S_{1}^{(1)}\right]$ can be extracted from the photon energy spectrum (四). The relative factor

$$
B_{F}=\frac{\sum_{a b c}\left(\frac{T_{F}}{2} d^{a b c}\right)\left(\frac{T_{F}}{2} d^{a b c}\right)}{\sum_{a b}\left(\frac{1}{2} \delta^{a b}\right)\left(\frac{1}{2} \delta^{a b}\right)}=\frac{N_{c}^{2}-4}{4 N_{c}} \stackrel{\left(N_{c}=3\right)}{=} \frac{5}{12}
$$

gives the ratio of the corresponding colour traces. Inclusive of the coupling constants the triple gluon energy spectrum in the decay $Q \bar{Q}\left[{ }^{3} S_{1}^{(1)}\right] \rightarrow g g g$ results in

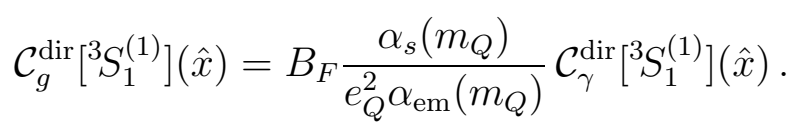

This formula contains a combinatorial factor $1 / 3=1 / 3$ ! : $1 / 2$ ! which compensates the aforementioned factor of three coming from the fact that all three final state gluons can fragment into a photon.

The colour octet coefficients $\mathcal{C}_{a}^{\text {dir }}\left[{ }^{2 S+1} L_{J}^{(8)}\right]$ are determined by the hard subprocesses in diagrams like fig. 2(b) and fig. 2(c). In both cases the kinematics are trivial. Thus we get for $\mathcal{C}_{g}^{\operatorname{dir}}[n]$ with $n \in\left\{{ }^{1} S_{0}^{(8)},{ }^{3} P_{0}^{(8)},{ }^{3} P_{2}^{(8)}\right\}$ from diagram $2(\mathrm{~b})$ :

$$
\mathcal{C}_{g}^{\operatorname{dir}}[n](\hat{x})=2 \frac{1}{2 \cdot 2 m_{Q}} \frac{1}{8 \pi} H[Q \bar{Q}[n] \rightarrow g g]\left(2 m_{Q}\right) \delta(1-\hat{x}) .
$$


Up to a factor of two which again is caused by the possibility for both gluons to fragment into a photon $\mathcal{C}_{g}^{\operatorname{dir}}[n](\hat{x})$ matches the corresponding differential decay modes $d \hat{\Gamma}_{n} / d \hat{x}$ without the NRQCD matrix element. The spin and colour averaged square of the amplitudes are [9]:

$$
\begin{aligned}
& H\left[Q \bar{Q}\left[{ }^{1} S_{0}^{(8)}\right] \rightarrow g g\right]\left(2 m_{Q}\right)=B_{F} \frac{128 \pi^{2} \alpha_{s}^{2}\left(m_{Q}\right)}{2 m_{Q}}, \\
& H\left[Q \bar{Q}\left[{ }^{3} P_{0}^{(8)}\right] \rightarrow g g\right]\left(2 m_{Q}\right)=B_{F} \frac{384 \pi^{2} \alpha_{s}^{2}\left(m_{Q}\right)}{2 m_{Q}}, \\
& H\left[Q \bar{Q}\left[{ }^{3} P_{2}^{(8)}\right] \rightarrow g g\right]\left(2 m_{Q}\right)=B_{F} \frac{512 \pi^{2} \alpha_{s}^{2}\left(m_{Q}\right)}{5 \cdot 2 m_{Q}} .
\end{aligned}
$$

Final states with $J=1$ are forbidden by the Landau-Yang theorem [30], i.e. $n={ }^{3} P_{1}^{(8)}$ and $n={ }^{3} S_{1}^{(8)}$ do not contribute to $Q \bar{Q}[n] \rightarrow g g$. Instead the latter configuration can decay into a light quark-antiquark pair (cf fig. 2(c)). The corresponding coefficient is

$$
\mathcal{C}_{a}^{\operatorname{dir}}\left[{ }^{3} S_{1}^{(8)}\right](\hat{x})=\frac{1}{2 \cdot 2 m_{Q}} \frac{1}{8 \pi} H\left[Q \bar{Q}\left[{ }^{3} S_{1}^{(8)}\right] \rightarrow q \bar{q}\right]\left(2 m_{Q}\right) \delta(1-\hat{x})
$$

where $a \in\{q, \bar{q}\}$ and

$$
H\left[Q \bar{Q}\left[{ }^{3} S_{1}^{(8)}\right] \rightarrow q \bar{q}\right]\left(2 m_{Q}\right)=\frac{64 \pi^{2} \alpha_{s}^{2}\left(m_{Q}\right)}{3 \cdot 2 m_{Q}} .
$$

Hence we have collected all the results for the partonic decay of a $Q \bar{Q}$ pair that are needed for the photon energy distribution in radiative decays of heavy quarkonia. Henceforth we will construct a model for shape functions in quarkonia decays and embed the partonic results into it.

\section{The shape function model}

In the following both direct and fragmentation contributions are improved by the application of the shape functions. We model these functions with the following physical picture in mind. According to the factorization assumption of NRQCD quarkonium decays are divided into two subprocesses as illustrated in fig. 3 . In the first stage the quarkonium $H$ radiates off a cluster of gluons with a momentum $k=\sum_{i} k_{i} \sim \mathcal{O}\left(m_{Q} v^{2}\right)$. The final state of this non-perturbative subprocess is a $Q \bar{Q}$ pair in the quantum state $n$ that annihilates perturbatively in the second stage. Its momentum is given by $p_{Q \bar{Q}}=P-\ell$ with $P^{2}=\left(2 m_{Q}\right)^{2}$, i.e. the non-perturbative momentum $\ell \sim \mathcal{O}\left(\Lambda_{\mathrm{QCD}}\right)$ measures the off-shellness of the quark-antiquark pair.

It is important to be careful with neglecting non-perturbative momenta in the hard subprocess because the light cone component $\ell_{+}$of the $Q \bar{Q}$ off-shellness is responsible for 


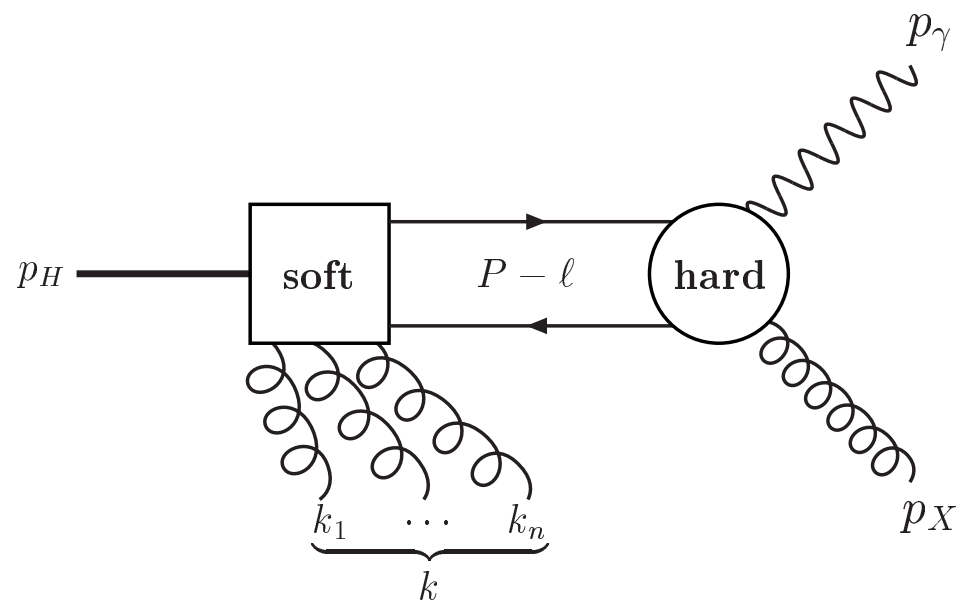

Figure 3: Schematic representation of direct colour octet contributions to the radiative quarkonium decay.

the shift of the partonic endpoint $m_{Q}$ to the physically correct hadronic value $E_{\gamma}^{\max }=M_{H} / 2$ [20]. Therefore we will keep the kinematics exactly here.

Nevertheless we have to model the radiation of the gluons from the initial state. This is done by our shape function [21]

$$
f_{n}^{H}(\ell)=\int \frac{d k^{2}}{2 \pi} \frac{d^{3} \boldsymbol{k}}{(2 \pi)^{3} 2 k^{0}}(2 \pi)^{4} \delta^{4}\left(p_{H}+k-P+\ell\right) \Phi_{n}\left(k ; p_{H}, P\right)
$$

where $\Phi_{n}$ is a radiator function parameterizing the emission of a soft gluon cluster with total momentum $k$. In our ansatz

$$
\Phi_{n}\left(k ; p_{H}, P\right)=a_{n} \cdot|\boldsymbol{k}|^{b_{n}} \exp \left\{-k_{0}^{2} / \Lambda_{n}^{2}\right\} \cdot k^{2} \exp \left\{-k^{2} / \Lambda_{n}^{2}\right\}
$$

the cut-off parameter $\Lambda_{n} \sim \mathcal{O}\left(m_{Q} v^{2}\right)$ reflect the expectation that the main contribution comes from the ultrasoft region where the energy $k_{0}$ and the invariant mass $k^{2}$ of the gluon cluster are of order $m_{Q} v^{2}$ and $\left(m_{Q} v^{2}\right)^{2}$ respectively. The choice

$$
\begin{aligned}
& b\left[{ }^{1} S_{0}^{(8)}\right]=2, \quad b\left[{ }^{3} P_{0}^{(8)}\right]=b\left[{ }^{3} S_{1}^{(8)}\right]=0, \\
& \Lambda\left[{ }^{1} S_{0}^{(8)}\right]=\Lambda\left[{ }^{3} P_{0}^{(8)}\right] \equiv \Lambda, \quad \Lambda\left[{ }^{3} S_{1}^{(8)}\right]=c \Lambda
\end{aligned}
$$

for the constants in (23) is motivated by the fact that the gluon coupling for a M1 magnetic dipole transition from the quarkonium $H$ to $Q \bar{Q}\left[{ }^{1} S_{0}^{(8)}\right]$ is proportional to the gluon momentum while an E1 or a double E1 electric dipole transition to $Q \bar{Q}\left[{ }^{3} P_{0,2}^{(8)}\right]$ or $Q \bar{Q}\left[{ }^{3} S_{1}^{(8)}\right]$ respectively does not have any $k$ dependence. Furthermore the necessity of at least two transitions for $n={ }^{3} S_{1}^{(8)}$ suggests the introduction of a factor $c=1.5$ to enlarge the average radiated energy and invariant mass in this case. Finally we fix $a_{n}$ by the normalization 
condition

$$
\int \frac{d^{4} \ell}{(2 \pi)^{4}} f_{n}^{H}(\ell)=\frac{1}{(2 \pi)^{3}} \int_{0}^{\infty} d k^{2} \int_{\sqrt{k^{2}}}^{\infty} d k_{0} \sqrt{k_{0}^{2}-k^{2}} \Phi_{n}\left(k ; p_{H}, P\right)=\left\langle H\left|\mathcal{O}_{n}\right| H\right\rangle .
$$

\subsection{Direct contributions}

After we have determined our model ansatz for the shape functions we proceed with the implementation of the hard subprocess. First we deal with the direct contributions. As mentioned above in leading order only the colour octet modes are interesting in the shape function formalism. Thus we start with the expression

$$
\begin{gathered}
d \Gamma_{8}^{\text {dir }}=\sum_{n} \int \frac{d^{4} \ell}{(2 \pi)^{4}} \frac{1}{2 M_{H}} \int{\widetilde{d p_{\gamma}}}_{\widetilde{d p}_{X}}(2 \pi)^{4} \delta^{4}\left(P-\ell-p_{\gamma}-p_{X}\right) H[Q \bar{Q}[n] \rightarrow \gamma g]\left(P, \ell, p_{\gamma}, p_{X}\right) \\
\cdot \int \frac{d k^{2}}{2 \pi} \frac{d^{3} \boldsymbol{k}}{(2 \pi)^{3} 2 k_{0}}(2 \pi)^{4} \delta^{4}\left(p_{H}-k-P+\ell\right) \Phi_{n}\left(k ; p_{H}, P\right)
\end{gathered}
$$

where one can easily recognize the shape function in the second line of the equation. In (26) $M_{H}$ denotes the quarkonium mass, $\widetilde{d p}_{\gamma}$ and $\widetilde{d p}_{X}$ the invariant phase space measures of the photon and the hard gluon respectively and finally the spin and colour averaged square $H_{n}$ of the hard subprocess amplitude is given by (5).

Manipulating eq. (26) we start with integration out the four momenta $k$ and $p_{X}$ with $p_{X}^{2}=0$ which determines the light cone components $\ell_{\perp}^{2}$ and $\ell_{+}$by the delta functions to

$$
\begin{aligned}
& \ell_{\perp}^{2}=\left(M_{H}-2 m_{Q}\right)\left(M_{H}-2 m_{Q}+2 \ell_{0}\right)+\ell_{+}\left(2 \ell_{0}-\ell_{+}\right)-k^{2}, \\
& \ell_{+}=\frac{1}{2 E_{\gamma}}\left[4 m_{Q}\left(M_{H}-E_{\gamma}\right)-M_{H}^{2}-2\left(M_{H}-2 E_{\gamma}\right) \ell_{0}+k^{2}\right] .
\end{aligned}
$$

To make use of these relations we also decompose $d^{4} \ell$ into its light cone components

$$
\int d^{4} \ell=\int_{0}^{2 \pi} d \phi \int d \ell_{0} d \ell_{+} \frac{d \ell_{\perp}^{2}}{2} \Theta\left(\ell_{\perp}^{2}\right)
$$

and rewrite the $\ell_{0}$ integration into a $k_{0}$ integration by $\ell_{0}=k_{0}-\left(M_{H}-2 m_{Q}\right)$. Then the integration over the azimuthal angular can be performed trivially because the partonic process is $\phi$ independent. Analogously the angular dependence in $\widetilde{d p}_{\gamma}$ is integrated out trivially and one gets $\widetilde{d p}_{\gamma}=E_{\gamma} d E_{\gamma} \Theta\left(E_{\gamma}\right) /\left(4 \pi^{2}\right)$. Finally we evaluate the equations (27). In simultaneous consideration of the theta function in (28) there arise integration bounds for $k_{0}$ where the physical ones are

$$
\frac{\left(M_{H}-2 E_{\gamma}\right)^{2}+k^{2}}{2\left(M_{H}-2 E_{\gamma}\right)} \leq k_{0} \leq \frac{M_{H}^{2}+k^{2}}{2 M_{H}}
$$


From this we can read off an upper bound for the $k^{2}$ integration

$$
k^{2} \leq M_{H}\left(M_{H}-2 E_{\gamma}\right)
$$

while the lower one is determined by $k^{2} \geq 0$. Introducing the abbreviations

$$
\alpha=\left(p_{H}-p_{\gamma}\right)_{+}=M_{H}-2 E_{\gamma}, \quad \beta=\left(p_{H}-p_{\gamma}\right)_{-}=M_{H}
$$

we finally end up with

$$
\frac{d \Gamma_{8}^{\mathrm{dir}}}{d E_{\gamma}}=\sum_{n} \int_{0}^{\alpha \beta} \frac{d k^{2}}{2 \pi} \int_{\left(\alpha^{2}+k^{2}\right) /(2 \alpha)}^{\left(\beta^{2}+k^{2}\right) /(2 \beta)} d k_{0} \frac{1}{2 M_{H}} \cdot \frac{1}{8 \pi} H[Q \bar{Q}[n] \rightarrow \gamma g]\left(M_{Q \bar{Q}}(k)\right) \cdot \frac{1}{4 \pi^{2}} \Phi_{n}\left(k ; p_{H}\right) .
$$

This is our master equation for the direct colour octet contributions to the photon energy spectrum that take into account shape functions effects within our model framework. Note that according to our model the partonic rate depends on

$$
M_{Q \bar{Q}}(k)=\sqrt{M_{H}^{2}-2 M_{H} k_{0}+k^{2}}
$$

rather than on $2 m_{Q}$, i.e. the quark mass in the partonic subprocess is effectively larger than $m_{Q}$. The colour singlet contributions are obtained by multiplying (3) with the colour singlet NRQCD matrix element $\left\langle H\left|\mathcal{O}_{1}\left({ }^{3} S_{1}\right)\right| H\right\rangle$. Here the heavy quark mass is set equal to $M_{H} / 2$.

\subsection{Fragmentation contribution}

The treatment of the fragmentation contributions is done in the following way: First we apply our shape function model on the colour octet contribution $d \hat{\Gamma} / d \hat{E}_{a}(Q \bar{Q}[n] \rightarrow a(\vec{a})$ to extend the reliability of the partonic NRQCD calculation up to higher values of the parton energy $\hat{E}_{a}$ in the $Q \bar{Q}$ rest frame. Afterwards we fold the received spectrum $d \Gamma / d E_{a}$ with the corresponding fragmentation function $D_{a \rightarrow \gamma}$ :

$$
\frac{d \Gamma_{8}^{\mathrm{frag}}}{d E_{\gamma}}=\sum_{a=g, q, \bar{q}} \int_{E_{\gamma}}^{M_{H} / 2} \frac{d E_{a}}{E_{a}} \frac{d \Gamma_{8}^{\mathrm{dir}}}{d E_{a}} D_{a \rightarrow \gamma}\left(E_{\gamma} / E_{a}\right)
$$

Note that the upper bound for the parton energy $E_{a}$ in the quarkonium rest frame is given by the hadronic value $M_{H} / 2$ rather than by the heavy quark mass $\hat{E}_{a}^{\max }=m_{Q}$ which defines the endpoint in the partonic calculation.

In (34) the folding of the gluon and the (anti)quark energy spectrum $d \Gamma_{8}^{d i r} / d E_{a}$ is calculated completely analogous to (32):

$$
\frac{d \Gamma_{8}^{\mathrm{dir}}}{d E_{a}}=\sum_{n} \int_{0}^{\bar{\alpha} \bar{\beta}} \frac{d k^{2}}{2 \pi} \int_{\left(\bar{\alpha}^{2}+k^{2}\right) /(2 \bar{\alpha})}^{\left(\bar{\beta}^{2}+k^{2}\right) /(2 \bar{\beta})} d k_{0} \frac{1}{2 M_{H}} \cdot \frac{1}{8 \pi} H[Q \bar{Q}[n] \rightarrow a \bar{a}]\left(M_{Q \bar{Q}}(k)\right) \cdot \frac{1}{4 \pi^{2}} \Phi_{n}\left(k ; p_{H}\right)
$$



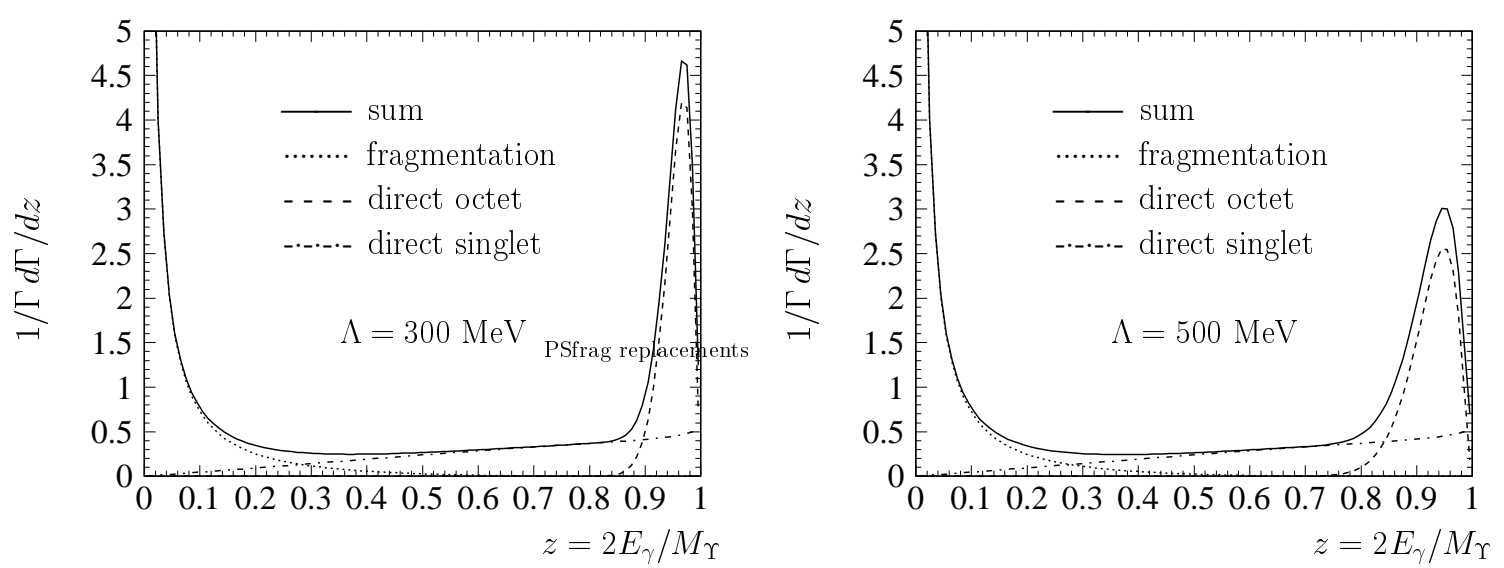

Figure 4: Photon energy spectrum in the radiative decay $\Upsilon(1 \mathrm{~S}) \rightarrow \gamma+$ light hadrons for two different values of the shape function model parameter: $\Lambda=300 \mathrm{MeV}$ (left) and $\Lambda=500 \mathrm{MeV}$ (right).

where the sum again runs only over the colour octet modes. $\bar{\alpha}$ and $\bar{\beta}$ are obtained from (31) by the substitution $E_{\gamma} \rightarrow E_{a}$. As described in subsection 2.2 the partonic subprocess $Q \bar{Q}[n] \rightarrow g g$ contributes only for $n={ }^{1} S_{0}^{(8)}, n={ }^{3} P_{0}^{(8)}$, and $n={ }^{3} P_{2}^{(8)}$ while $n={ }^{3} S_{1}^{(8)}$ needs a light quark-antiquark pair in the final state. The sum in (34) runs not only over the gluon but also over different (anti)quark flavours $q=\{u, d, s(, c)\}$ where we additionally assume $D_{q \rightarrow \gamma}(\xi)=D_{\bar{q} \rightarrow \gamma}(\xi)$.

\section{Results}

As mentioned above we restrict our numerical analysis on the bottomonium system since the fragmentation contributions in the charmonium sector are not very trustworthy within our approach. For the photon spectrum in the radiative decay of a $\Upsilon(1 \mathrm{~S})$ we choose the following set of parameters: $M_{\Upsilon}=9.46 \mathrm{GeV}, \alpha_{s}\left(\mu^{2}\right)=0.190, \alpha_{\mathrm{em}}\left(\mu^{2}\right)=1 / 132$ where the factorization scale $\mu$ is fixed at the $b$ quark mass $m_{b}=4.8 \mathrm{GeV}$. Unfortunately the values of the NRQCD matrix elements are unknown. While the production matrix elements of the bottomonium sector have been fitted in a recent analysis by Braaten, Fleming and Leibovich [6] we have to make do with the NRQCD scaling rules

$$
\left\langle\Upsilon\left|\mathcal{O}_{8}\left({ }^{1} S_{0}\right)\right| \Upsilon\right\rangle \sim \frac{\left\langle\Upsilon\left|\mathcal{O}_{8}\left({ }^{3} P_{0}\right)\right| \Upsilon\right\rangle}{m_{b}^{2}} \sim\left\langle\Upsilon\left|\mathcal{O}_{8}\left({ }^{3} S_{1}\right)\right| \Upsilon\right\rangle \sim v^{4}\left\langle\Upsilon\left|\mathcal{O}_{1}\left({ }^{3} S_{1}\right)\right| \Upsilon\right\rangle
$$

to estimate at least the order of magnitude of the decay matrix elements needed for the normalization, i.e. for the relative weights, of the different contributing channels. For our numerical evaluation we take for each colour octet matrix element $2.20 \cdot 10^{-2} \mathrm{GeV}^{2}$ and $\left\langle\Upsilon\left|\mathcal{O}_{1}\left({ }^{3} S_{1}\right)\right| \Upsilon\right\rangle=3.43 \mathrm{GeV}^{2}$, i.e. we set $v^{2}=0.08$ for bottomonia. Finally we normalize the total rate to one. In case of a comparison with data this could be changed easily.

The result for two different values of our shape function model parameter $\Lambda \sim m_{b} v^{2}$ is show in fig. $⿴$. One recognizes that fragmentation contributions are non-negligible for small photon energies only. In the region $0.1 \lesssim z \lesssim 0.4$ they are mainly dominated by the 

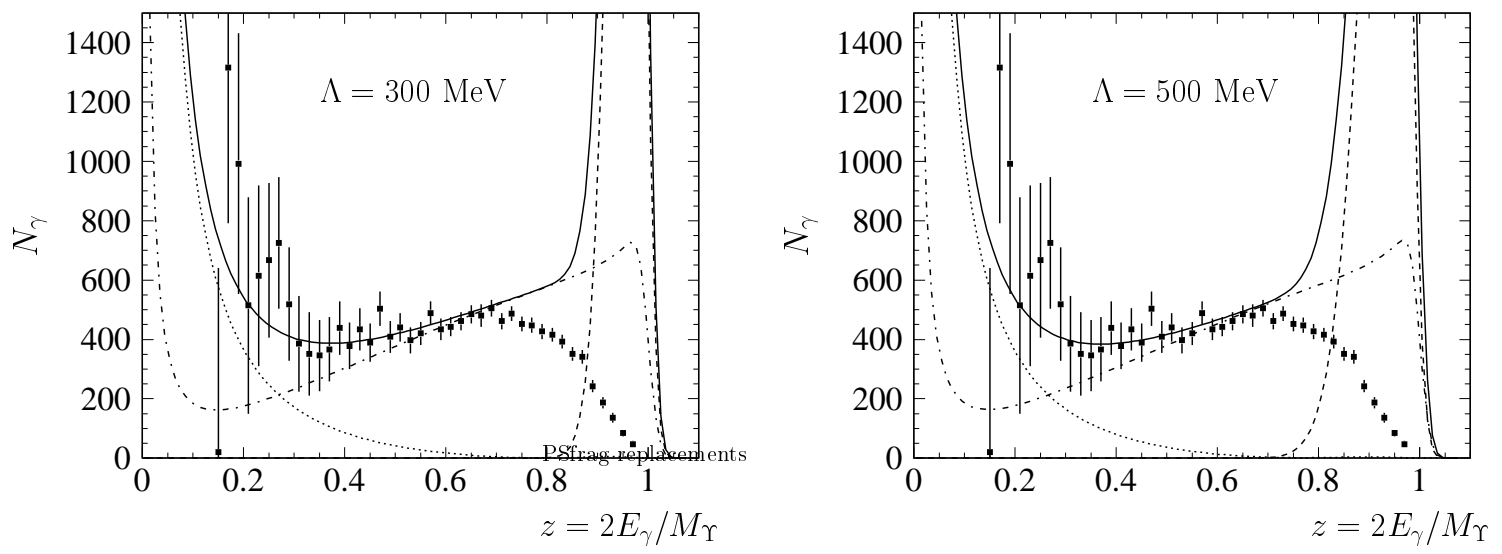

Figure 5: Comparison of the theoretical spectrum (solid line) for $\Lambda=300 \mathrm{MeV}$ (left) and $\Lambda=500 \mathrm{MeV}$ (right) with data of CLEO [17]. The dashed line shows the direct, the dotted line the fragmentation contribution. The spectrum predicted by the colour singlet model (direct + fragmentation, without any hadronization model) is indicated by the dash-dotted curvature.

${ }^{3} S_{1}^{(8)}$ channel. Though this channel is connected with the fragmentation function $D_{q \rightarrow \gamma}(\xi)$ which diverges for $\xi \rightarrow 1$ its numerical contribution to the spectrum for large values of $z$ is negligible. This matches to our expectation that fragmentation processes prefer to transfer small energy fractions from the gluon (quark) to the photon.

The upper endpoint region $z \gtrsim 0.8$ is dominated by direct colour octet contributions (at least in leading order $\left.\alpha_{s}\right)$. While the partonic result is proportional to $\delta(1-\hat{z})$ the soft gluon radiation in the initial state smears out the delta peak and also shifts its maximum to $z_{Q \bar{Q}}=$ $M_{Q \bar{Q}}^{\text {eff }} / M_{\Upsilon}<1$. The higher the model parameter $\Lambda$ is the smaller is $z_{Q \bar{Q}}$ and therewith the effective heavy quark mass and the broader is the width of the peak. Comparing the integrated rates of the direct colour singlet mode ${ }^{3} S_{1}^{(1)}$ and the direct colour octet ones we realize that their contributions are almost equal. This can be explained by the fact that the suppression factor $v^{4}=6.4 \cdot 10^{-3}$ of the colour octet modes is canceled by an additional factor $\alpha_{s}\left(m_{b}\right) /(4 \pi)=1.5 \cdot 10^{-2}$ in the colour singlet mode (cf equation (1)). Furthermore one have to consider that there are contributions from many colour octet modes $\left({ }^{2 S+1} L_{J}^{(C)}={ }^{1} S_{0}^{(8)},{ }^{3} S_{1}^{(8)},{ }^{3} P_{0}^{(8)}\right.$ and $\left.{ }^{3} P_{2}^{(8)}\right)$ but only from one colour singlet mode $\left({ }^{3} S_{1}^{(1)}\right)$.

Let us finally concentrate on the region of middle high photon energies. From the theoretical point of view the part between $0.4 \lesssim z \lesssim 0.75$ is the cleanest one of the spectrum. Here the colour singlet contribution which is assumed to be dominating over the whole photon energy range in the colour singlet model can be extracted within shape function improved NRQCD without any pollution from other channels. This is still true after including the next-to-leading order contributions: While the colour singlet corrections steepen the slope [8] the colour octet terms are negligible for $0.4 \lesssim z \lesssim 0.75$ [9].

Since the extraction of $\alpha_{s}\left(m_{b}\right)$ needs an extrapolation of the measured spectrum towards $z=0$, a complete theoretical understanding of the part of the spectrum the fit is based 
on is indispensable to reach an accurate value for the strong coupling constant. In our opinion this is not possible for high photon energies. To illustrate the problems in the upper endpoint region we smear out our theoretical result with the energy resolution of the CLEO detector

$$
\frac{\sigma_{E}}{E}(\%)=\frac{0.35}{E^{0.75}}+1.9-0.1 E
$$

and fit it to their most recent data [17] for $0.4 \leq z \leq 0.7$. The result is shown in fig. 5. While the spectrum is described satisfactorily within the fragmentation uncertainties for small and middle high values of $z$ the discrepancy between theory and experiment is overwhelming for $z \gtrsim 0.75$. As shown by the CLEO collaboration [17] the CSM result combined with the fragmentation contributions according to [12] can be brought into agreement with data using a hadronization model by Field [13] even though the consequential value of $\alpha_{s}\left(m_{b}\right)$ is slightly to small compared to the one measured on the $Z_{0}$ resonance.

Although the colour octet contributions in fig. 5 have not been suppressed by a hadronization model yet, they seem to be in strong contradiction to the experimental observation. The simplest explanation for this deviation would be an extreme smallness of the colour octet NRQCD decay matrix elements even smaller than the $v^{4}$ suppression still acknowledged by the power counting rules. Some hints for such small colour octet decay matrix elements also come from the estimation of the $\alpha_{s}$ corrections [9]. Furthermore a recent analysis of the corresponding production matrix elements [6] yielded smaller values than predicted by velocity scaling rules, too. Although the crossing symmetry between these production matrix elements and the ones of the decay holds only in leading order perturbation theory this could also be interpreted as indication for somehow suppressed (or even negative?) values of the NRQCD matrix elements.

Nevertheless the understanding of the upper endpoint region in the radiative decay of the $\Upsilon(1 \mathrm{~S})$ is not good enough to conclude convincingly that the colour octet matrix elements are extremely small. Without having investigated the Sudakov corrections on the colour octet contributions and without a better understanding of the hadronization process it seems impossible to give a stringent theoretical prediction for $z \gtrsim 0.75$. Furthermore the experimental investigation of this spectrum suffers from large systematic problems in this kinematical regime, too.

In summary an extraction of a precise value for the strong coupling constant from the radiative $\Upsilon$ decay seems to be impossible as long the upper endpoint region is included in the fit. Unless both theoretical and experimental progress concerning the physics in the upper endpoint region is achieved $\alpha_{s}\left(m_{b}\right)$ should be fitted from the data between $0.4 \leq z \leq 0.7$ only.

\section{Acknowledgements}

I would like to thank M. Beneke for useful comments and for reading the manuscript. The author is supported by the Graduiertenkolleg "Elementarteilchenphysik an Beschleunigern" and the DFG-Forschergruppe "Quantenfeldtheorie, Computeralgebra und Monte-CarloSimulation". 


\section{References}

[1] J.J. Aubert et al., Phys. Rev. Lett. 33 (1974) 1404,

J.E. Augustin et al., Phys. Rev. Lett. 33 (1974) 1406.

[2] S. W. Herb et al., Phys. Rev. Lett. 39 (1977) 252.

[3] T. Appelquist and H. D. Politzer, Phys. Rev. Lett. 34 (1975) 43.

[4] S. J. Brodsky, D. G. Coyne, T. A. DeGrand and R. R. Horgan, Phys. Lett. B73 (1978) 203.

[5] G.T. Bodwin, E. Braaten and G.P. Lepage, Phys. Rev. D51 (1995) 1125,

Erratum: Phys. Rev. D55 (1997) 5853.

[6] E. Braaten, S. Fleming and A. K. Leibovich, hep-ph/0008091.

[7] G. T. Bodwin, D. K. Sinclair and S. Kim, Phys. Rev. Lett. 77 (1996) 2376.

[8] M. Krämer, Phys. Rev. D60 (1999) 111503.

[9] F. Maltoni and A. Petrelli, Phys. Rev. D59 (1999) 074006.

[10] D. M. Photiadis, Phys. Lett. B164 (1985) 160.

[11] F. Hautmann, Proceedings of International Conference on the Structure and the Interactions of the Photon (Photon 97) including the 11th International Workshop on Photon-Photon Collisions, Egmond aan Zee, Netherlands, 10-15 May 1997, hep$\mathrm{ph} / 9708496$.

[12] S. Catani and F. Hautmann, Nucl. Phys. Proc. Suppl. 39BC (1995) 359.

[13] R. D. Field, Phys. Lett. B133 (1983) 248.

[14] G. Parisi and R. Petronzio, Phys. Lett. B94 (1980) 51.

[15] G. S. Abrams et al., Phys. Rev. Lett. 44 (1980) 114, M. T. Ronan et al., Phys. Rev. Lett. 44 (1980) 367,

D. L. Scharre et al., Phys. Rev. D23 (1981) 43.

[16] R. D. Schamberger et al., Phys. Lett. B138 (1984) 225,

S. E. Csorna et al. [CLEO Collaboration], Phys. Rev. Lett. 56 (1986) 1222,

H. Albrecht et al. [ARGUS Collaboration], Phys. Lett. B199 (1987) 291,

A. Bizzeti et al. [Crystal Ball Collaboration], Phys. Lett. B267 (1991) 286.

[17] B. Nemati et al. [CLEO Collaboration], Phys. Rev. D55 (1997) 5273.

[18] M. Consoli and J. H. Field, Phys. Rev. D49 (1994) 1293,

M. Consoli and J. H. Field, J. Phys. G G23 (1997) 41. 
[19] T. Mannel and S. Wolf, TTP97-02, hep-ph/9701324.

[20] I.Z. Rothstein and M.B. Wise, Phys. Lett. B402 (1997) 346.

[21] M. Beneke, G. A. Schuler and S. Wolf, Phys. Rev. D62 (2000) 034004.

[22] M. Beneke, I.Z. Rothstein and M.B. Wise, Phys. Lett. B408 (1997) 373.

[23] J. F. Owens, Rev. Mod. Phys. 59 (1987) 465.

[24] P. Aurenche, P. Chiappetta, M. Fontannaz, J. P. Guillet and E. Pilon, Nucl. Phys. B399 (1993) 34, M. Glück, E. Reya and A. Vogt, Phys. Rev. D48 (1993) 116, Erratum: Phys. Rev. D51 (1995) 1427, L. Bourhis, M. Fontannaz and J. P. Guillet, Eur. Phys. J. C2 (1998) 529.

[25] V. N. Gribov and L. N. Lipatov, Yad. Fiz. 15 (1972) 781, Sov. Phys. JETP 15 (1972) 438 , V. N. Gribov and L. N. Lipatov, Yad. Fiz. 15 (1972) 1218, Sov. Phys. JETP 15 (1972) 675 ,

L. N. Lipatov, Yad. Fiz. 20 (1974) 181, Sov. J. Nucl. Phys. 20 (1975) 94,

Y. L. Dokshitzer, Zh. Eksp. Teor. Fiz. 73 (1977) 1216, Sov. Phys. JETP 46 (1977) 641.

[26] G. Altarelli and G. Parisi, Nucl. Phys. B126 (1977) 298.

[27] E. W. Glover and A. G. Morgan, Z. Phys. C62 (1994) 311.

[28] D. Buskulic et al. [ALEPH Collaboration], Z. Phys. C69 (1996) 365.

[29] A. Ore and J. L. Powell, Phys. Rev. 75 (1949) 1696.

[30] L. D. Landau, Sov. Phys. Dokl. 60 (1948) 207, C. N. Yang, Phys. Rev. 77 (1950) 242. 